\title{
Bank Notes Authentication System based on Wavelet Features and Artificial Neural Network
}

\author{
Ch.Ratna Jyothi \\ Reaearch scholar, Dept of \\ computer science,Krishna \\ university,Machilipatnam
}

\author{
Y.K. Sundara Krishna, \\ PhD \\ Professor and Dean, Dept of \\ computer science, Krishna \\ University, Machilipatnam
}

\author{
V. Srinivasa Rao, PhD \\ Professor\&Head, Dept of \\ computer science and engg, \\ VRSEC,Vijayawada
}

\begin{abstract}
Monetary transactions are integral part of our day to day activities, so currency authentication has become one of the active research area at present and it has vast potential applications. In this paper we introduced a system to verify the authentication of banknotes bench mark dataset using computer vision. We considered 1372 samples of various bank notes in our work. The technology of currency authentication aims to search and extract potential features of paper currency for efficient classification. Features were extracted from images that were taken from genuine and forged banknote-like specimens. They are classified using Artificial Neural network. The variance of Wavelet Transformed image (continuous), skewness of Wavelet Transformed image (continuous), curtosis of Wavelet Transformed image (continuous),entropy of image (continuous) features are extracted from images for accurate classification. Our proposed system able to authenticate with zero percent misclassification.
\end{abstract}

\section{General Terms}

Pattern Recognition,Authentication

\section{Keywords}

Currency recognition, Artificial Neural Network,

Classification,wavelets

\section{INTRODUCTION}

Object recognition [1-7] and authentication is an important and highly demanded area of pattern recognition. An object can be anything in real life. It can be text in a document, a license plate of a vehicle, an iris in a person's eyes, a sign in a sign language, a face of a person, and so on. Similarly, paper currency recognition [8-15,17-20] is as important as any other object recognition.

Some authors, in the recent years, have contributed to the subject of paper currency recognition systems. These existing paper currency recognition methods, in the literature, mainly involve image processing and/or neural network techniques [11-15].

This paper deals with a simple, efficient and very accurate approach in the system design. In designing such a system, it considers different dimensions, Color luminance, Edge histogram using max sobel gradient, correlations as features. A different method using radial basis Function networks, is utilized for developing an intelligent system which can recognize paper currency. This research is specifically designed for recognizing paper currency from United Kingdom ("Pound"), Japan ("Yen"),Europe("euro") and
India("rupee"). In the proposed paper recognition technique has been designed in such a way that it can be used for recognizing paper currency of four different countries.. To overcome the problem of recognizing dirty banknotes, the pre-processing stage is also considered.

The proposed scheme is different from various existing methods [1-21] because of its approaches in the recognition phases. Specifically, for example, sobel masks have been used in ${ }^{11}$ for considering specific edges (images) in a paper currency. Using this method, the features are extracted and fed to a Neural Network (NN). The technique in [20] deals with Pakistani paper currency with very different feature set which is specific to regional currency marks and color of the currency. Similarly, the technique introduced in $^{21}$ is different from the proposed technique as it introduces much more number of features than the ones introduced in the proposed method.

The organization of the paper is as follows. Section 2 introduces the overall mechanism for PCR, In Section 3, the pre-processing steps are briefly introduced. Section 4 describes the problem formulation for four different currencies. The proposed PCR approach, together with feature extraction method as well as classification has been completely discussed in Section 5. Section 6 describes details of experimental results for the case of multiple Paper Currency. Finally, Section 7 concludes the paper.

\section{STRUCTURE OF TYPICAL CURRENCY AUTHENTICATION SYSTEM}

The system presented is designed to recognize paper currency. Input to the system is an image acquired by a scanner or a digital camera, containing the paper currency and its output is the features of the paper currency. The system consists of the modules: Image acquisition, pre-processing including noise removal, feature extraction, classification and recognition. The structure of the system is shown in Fig. 1.

\section{PRE-PROCESSING}

In the proposed system a high resolution scanner is used to acquire the image. The acquired image of a paper currency is first converted to gray scaled image. Conversion to gray scale facilitates further pre-processing. The task of pre-processing is achieved by converting colored currency images into grayscale, then black-white images. After that, the edge of the image is filtered using Prewitt method. Then, the image edge is detected using Canny' s edge detection method. Different currency images are shown in Fig. 2.

This paper is meant for Paper Currency Authentication System. It is designed to just authenticate different 
currencies. This means that this system will not be concerned with currencies other currencies. In addition, the system will focus on paper currencies, not coins. Moreover, the system is concerned with verification of the validity of the paper currencies (i.e. verifying that the paper currency is genuine and not faked). This is usually done using other methods which might involve sensing the magnetic string embedded inside the currency, or some other methods.

The goal of the paper is to achieve the best accuracy in recognizing patterns with the lowest possible. Given the fact that paper currencies are usually recognized by machines that have small power (such as auto-seller machines and ATM's), the cost is a limiting factor. Therefore, it is really urgent for all paper currency recognizers to minimize the power consumption, and, at the same time, achieve high level of accuracy.

\section{PROPOSED APPROACH}

\subsection{Image Acquisition}

In this step we scan images of four different currencies of good quality and bad quality for training as well as for testing purpose.

\subsection{Image Preprocessing}

Median filter is used for de noising the image after resizing the image. A median filter considers each pixel at the same time and looks at its neighboring pixels to decide whether it is representative of its surroundings or not. The pixel value is replaced with median of neighboring pixel value instead of replacing with mean. Median filter is more effective when the goal is to simultaneously reduce noise and at the same time to preserve edges in the image. From the input color image each Red, Blue and Green channels are denoised separately by using median filter.

\subsection{Feature Extraction}

In this section we present an approach to extract the features of currency notes. The features extracted constitute input vector to the system. The potential features are extracted for efficient classification. The input vector can be written as follows:

$\mathrm{F}=(\mathrm{V}, \mathrm{S}, \mathrm{C}, \mathrm{E})$

Which consists of features as its arguments, shown in Table1

Features were extracted from images that were taken from genuine and forged banknote-like specimens. The attributes of the images such as The variance of Wavelet Transformed image (continuous), skewness of Wavelet Transformed image (continuous),curtosis of Wavelet Transformed image (continuous),entropy of image (continuous) features are extracted from images for accurate classification.

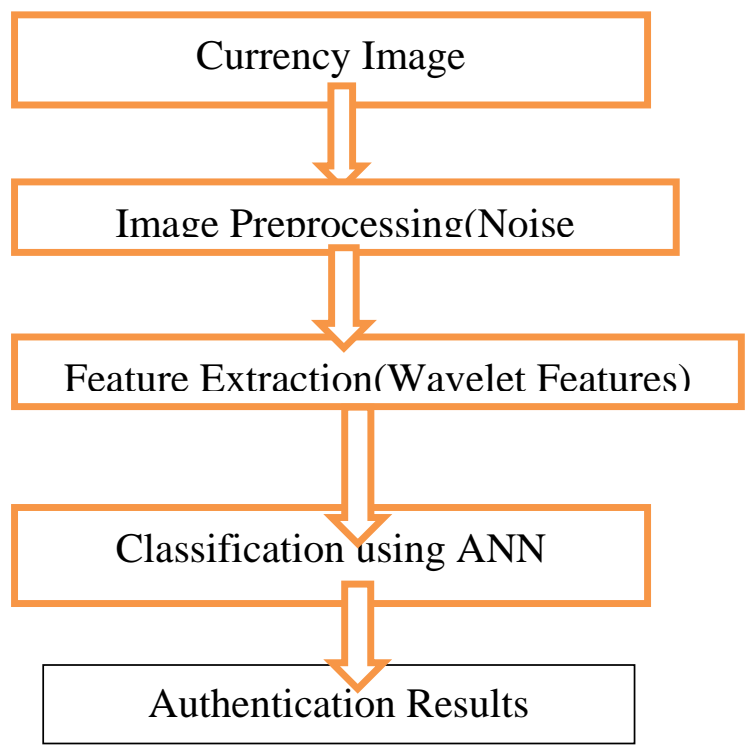

Fig.1 Flow chart of Currency authentication System

Table 1.List of features in the input feature vector

\begin{tabular}{|c|l|l|}
\hline$\#$ & $\begin{array}{l}\text { Feature } \\
\text { Notation }\end{array}$ & Feature Specification \\
\hline 1 & $V$ & variance of Wavelet Transformed image \\
\hline 2 & $S$ & skewness of Wavelet Transformed image \\
\hline
\end{tabular}




\begin{tabular}{|l|l|l|}
\hline 3 & $C$ & curtosis of Wavelet Transformed image \\
\hline 4 & $E$ & entropy of image \\
& & \\
\hline
\end{tabular}

\section{ClASSIFICATION ALGORITHM}

Once the features are extracted it is essential to classify the currency based on these features by using an effective currency authentication system called classifier. One of the most common classifier which had been used recently is Artificial neural network .A neural network based recognition schemes are used by many researchers in the literature $[9,10,11,12]$.

\subsection{Training Phase}

Initially the feedforward backpropagation neural network is trained with 1372 samples of from genuine and forged banknote-like specimens. The performance and training state results are shown below in fig $3 \&$ fig 4 respectively. Feed forward backpropagation neural network originate from the problem of interpolating a set of data in a hyperspace to find the best hyper-plane that interpolates that set of data. In feedforward backpropagation Neural Networks, there are three layers: input layer, hidden layer and output layer. The input layer consists of the data (or the pattern) that is to be interpolated (or classified). The hidden layer provides a nonlinear transformation from the input layer space to the hidden layer space. It usually consists of a high number of neurons. The output layer provides a linear transformation from the hidden layer space to the output layer space ${ }^{6}$. The network is trained with 100 images which contains normal, noisy and tilted images.

\subsection{Testing Phase}

Once the network has been trained on different currencies with the their corresponding feature vectors along with target class and the trained network is validated against test images to check the performance of the proposed system

\section{EXPERIMENTAL RESULTS}

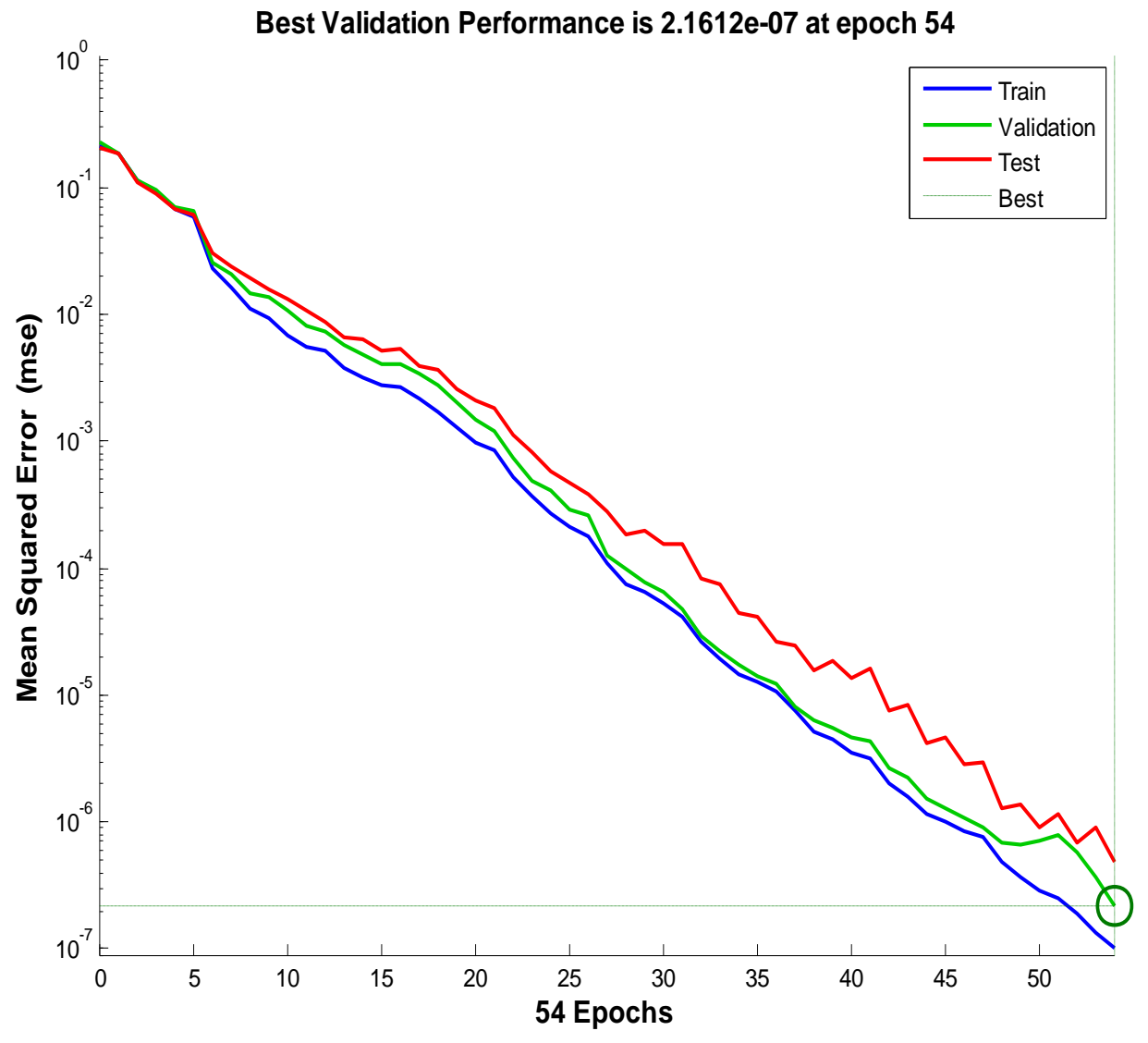

Fig 2: Performance of network classifier 

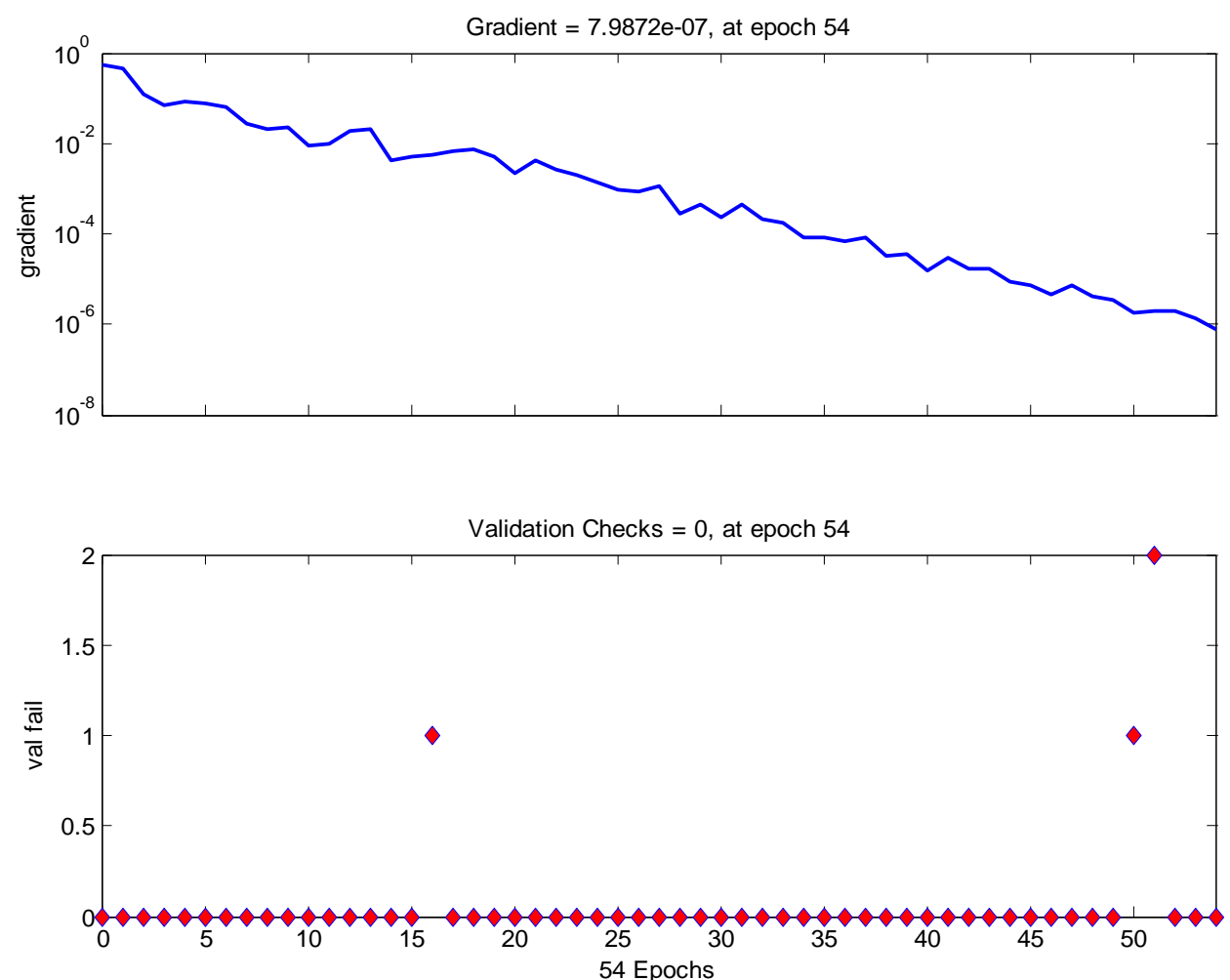

Fig 3 Training State of the network classifier

Table 3. Experimental Results of feedforward back propagation Network Classifier.

\begin{tabular}{|l|l|l|l|}
\hline & \multicolumn{1}{|c|}{ Samples } & MSE(Mean Square Error) & \%Error \\
\hline Training & 960 & $1.03 \mathrm{E}-07$ & 0 \\
\hline Validation & 206 & $2.16 \mathrm{E}-07$ & 0 \\
\hline Testing & 206 & $4.79 \mathrm{E}-07$ & 0 \\
\hline
\end{tabular}

The recognition results are as shown in Table 3.From the table it can observed that our system achieved zero percent error means zero miscalssifications.

\section{CONCLUSION AND FUTURE WORK}

Paper Currency Recognition is an important application of Pattern Recognition. Many studies were made to recognize paper currencies using Neural Networks. In this paper, another method of recognizing currencies has been introduced, which is based wavelet features of the images. The method uses feed forwardd backpropagation neural network. The method is quite reasonable in terms of accuracy. However, there is a room to improve the processing time.

The proposed algorithm is fully automatic and requires no human intervention. The author is also thinking to apply the proposed feature methodology for another model of classification. It might improve the accuracy and efficiency process.

\section{REFERENCES}

[1] Hassanpour H, Farahabadi, PM. Using Hidden Markov Models for paper currency recognition, Expert Systems with Applications 2009; 36(6): 10105-10111.

[2] Gonzalez RC, Woods RE. Digital image processing, Prentice Hall; 2009.
[3] Hassanpour H, Mesbah M. Neonatal EEG seizure detection using spike signatures in the time-frequency domain, IJE Transactions A: Basics2007; 20(2):137-146.

[4] Hassanpour H, Yaseri A, Ardeshir G. Feature Extraction for Paper currency Recognition. In International symposium on signal processing and its applications (ISSPA), Sharjah, UAE; 2007.

[5] Iosifescu M. Finite Markov process and their applications. New York, NY: Wiley; 1980.

[6] Jae LS. Two-dimensional signal and image processing. Englewood Cliffs, NJ: Prentice Hall; 1990.

[7] Kim M, Kim D, Lee S. Face recognition using the embedded HMM with second-order block-specific observations. Pattern Recognition2003; 36(11):27232733.

[8] Takeda F, Nishikage T. Multiple kinds of paper currency recognition using neural network and application for Euro currency. In IEEE International Joint Conference on Neural Networks, Como, p. 143-147; 2000.

[9] Toussaint GT. Proximity graphs for nearest neighbor decision rules: Recent progress. Proceedings of the $34^{\text {th }}$ Symposium on the INTERFACE, p. 17-20; 2002. 
[10] Vila A, Ferrer N, Mantecon J, Breton D, Garcia, JF. Development of a fast and non-destructive procedure original and fake euro notes.Analytica Chimica Acta 2006; 559:257-263.

[11] Zhang EH, Jiang B, Duan JH, Bian ZZ. Research on paper currency recognition by neural networks. In Proceeding of the second international conference on machine learning and cybernetics, p. 2193-2197; 2003.

[12] Liu Q, Tang L. Study of Printing Identification Based on Multi-spectrum Imaging Analysis, Proceedings of the International Conference on Computer Science and Software Engineering, p. 229 - 232; 2008.

[13] Ahmadi A, Omatu S, Yoshioka M. Implementing a Reliable Neuro-Classifier for Paper Currency Using PCA Algorithm, Proceedings of the $41^{\text {st }}$ SICE Annual Conference, p. 2466-2488; 2002.

[14] Takeda F, Omatu S. High Speed Paper Currency Recognition by Neural Networks, IEEE Transactions on Neural Networks 1995; 6(1):73-77.

[15] Takeda F, Omatu S, Onami S, Kadono T, Terada K. A Paper Currency Recognition Method by a Small Size Neural Network with OptimizedMasks by GA,
Proceedings of the IEEE World Congress on Computational Intelligence, Orlando, USA, 7, p.42434246; 1994

[16] Bow, Sing-Tze. Pattern Recognition and Image Preprocessing. New York: Marcel Dekker, Inc., 2002.

[17] Hasanuzzaman FM, Yang X, Tian YL. Robust and effective component-based banknote recognition by SURF features, Proceedings of the20th Annual Wireless and Optical Communications Conference, p. 1-6; 2011.

[18] He J, Hu Z, Xu P, Jin O, Peng M. The design and implementation of an embedded paper currency characteristic data acquisition system, Proceedings of the International Conference on Information and Automation, p. 1021-024; 2008

[19] Junfang G, Yanyun Z, Anni C. A reliable method for paper currency recognition based on LBP, Proceedings of the 2nd IEEE InternationalConference on Network Infrastructure and Digital Content, p. 359-363; 2010.

[20] Sargano AB, Sarfraz M, Haq N. An Intelligent System for Paper Currency Recognition with RobustFeatures, Journal of Intelligent and Fuzzy Systems 2014; 27(4): $1905-1913$ 